\title{
Diabetes and Metabolism
}

\section{Fasting and postchallenge hyperglycemia and risk of cardiovascular disease in Chinese: The Chin-Shan Community Cardiovascular Cohort study}

Kuo-Liong Chien, MD, PhD, a,b,c Hsiu-Ching Hsu, PhD, ${ }^{\text {b }}$ Ta-Chen Su, MD, PhD, ${ }^{\text {b }}$ Ming-Fong Chen, MD, PhD, Yuan-Teh Lee, MD, PhD, ${ }^{b}$ and Frank B. Hu, MD, PHD ${ }^{\mathrm{a}}$ Boston, MA; and Taipei, Taiwan

\begin{abstract}
Background Whether fasting glucose is superior to postchallenge glucose or insulin level for prediction of cardiovascular disease (CVD) remains controversial. The aim of our study was to compare fasting, postchallenge glucose and other markers as predictors of CVD in a community-based prospective cohort study among 2,165 adult participants.
\end{abstract}

Methods A standard 75-g oral glucose tolerance test was performed, with measurements of fasting and 2-hour postchallenge plasma glucose and insulin levels. We defined the CVD outcome as incident coronary heart disease and stroke. Cox regression model was used to estimate the relative risk (RR) for CVD.

Results A total of 166 individuals developed major CVD events during 10.5 years of follow-up. Both fasting and postchallenge glucose were significantly associated with CVD risk (adjusted RR in the highest quartile vs the lowest quartile $1.74,95 \%$ confidence interval $[\mathrm{Cl}] 1.06-2.86$ for fasting glucose; RR in highest quartile $2.05,95 \% \mathrm{Cl} 1.23-3.42$ for postchallenge glucose). Postchallenge and fasting glucose had similar areas of receiver operative characteristics curves (0.65, $95 \% \mathrm{Cl}$ 0.58-0.72 for postchallenge glucose; $0.65,95 \% \mathrm{Cl}$ 0.58-0.72 for fasting glucose). In mutually adjusted models, fasting and postchallenge glucose remained significant risk factors for CVD, whereas insulin resistance variables became nonsigificant.

Conclusions These findings show that fasting and postchallenge glucose concentrations are independent predictors of CVD risk among ethnic Chinese in Taiwan. (Am Heart J 2008;156:996-1002.)

Abnormal glucose homeostasis, presented by fasting hyperglycemia or postchallenge hyperglycemia, is a precursor to diabetes mellitus and is widely used as diagnostic criteria for diabetes. ${ }^{1,2}$ Postchallenge hyperglycemia, a marker for impaired glucose tolerance, is also an indicator for peripheral insulin resistance. ${ }^{2,3}$ Fasting hyperglycemia, with elevated fasting glucose level, can result from elevated hepatic glucose output and/or defect in early insulin secretion. ${ }^{2,4}$ Although both postchallenge and fasting hyperglycemia are considered as important risk factors for cardiovascular disease (CVD) end points, few prospective studies have compared the relationships

From the a Department of Nutrition, School of Public Health, Harvard University, Boston, MA, bepartment of Internal Medicine, National Taiwan University Hospital, Taipei, Taiwan, and 'Institute of Preventive Medicine, College of Public Health, National Taiwan University, Taipei, Taiwan.

Doctor Frank Hu's research is partly supported by the American Heart Association Established Investigator Award.

Submitted August 1, 2007; accepted June 16, 2008.

Reprint requests: Yuan-Teh Lee, MD, PhD, Department of Internal Medicine, National Taiwan University Hospital, Taipei 100, Taiwan. Frank B. Hu, MD, PHD, Department of Nutrition, Harvard School of Public Health, Harvard University, Boston, MA 02115. E-mails: ytlee@ha.mc.ntu.edu.tw, frank.hu@channing.harvard.edu

0002-8703/\$ - see front matter

(C) 2008, Mosby, Inc. All rights reserved.

doi:10.1016/i.ahj.2008.06.019 between these variables and risk of CVD. ${ }^{2}$ Insulin resistance markers, including homeostasis model assessment (HOMA), ${ }^{5}$ quantitative insulin sensitivity check index (QUICKI), ${ }^{6}$ and insulin sensitivity index $\left(\mathrm{ISI}_{0,120}\right),{ }^{7}$ have also been associated with cardiovascular risk, ${ }^{8}$ but data are sparse in Asian populations. Therefore, we conducted a prospective study to examine the roles of postchallenge glucose and other insulin resistance biomarkers in predicting CVD incidence among ethnic Chinese in Taiwan.

\section{Subjects and methods}

Study participants

Details of this cohort study have been published previously. ${ }^{9-11}$ Briefly, the Chin-Shan Community Cardiovascular Cohort (CCCC) study began in 1990 by recruiting 1,703 men and 1,899 women aged $\geq 35$ years in the Chin-Shan township. Information about anthropometry, lifestyle, and medical conditions was assessed by interview questionnaires in 2-year cycles, and the validity and reproducibility of the collected data and measurements have been reported in detail elsewhere. ${ }^{10}$ The participants were invited to receive $75 \mathrm{~g}$ glucose tolerance test during the survey in 1994-1995. ${ }^{11}$ For follow-up cohort 
Table I. Distribution of baseline demographic, lifestyle, and socioeconomic factors in the Study Population in the CCCC cohort (1994-1995), specified by fasting and postchallenge glucose quartiles

\begin{tabular}{|c|c|c|c|c|c|c|c|c|c|c|}
\hline & & & Fasting & & & & Pos & stchallenge & & \\
\hline & 1 & 2 & 3 & 4 & & 1 & 2 & 3 & 4 & \\
\hline & $<97$ & 97-104 & $105-117$ & $\geq 118$ & & $<101$ & $101-122$ & 123-152 & $\geq 153$ & \\
\hline & $n=584$ & $n=508$ & $n=556$ & $n=516$ & $\boldsymbol{P}$ & $n=542$ & $n=543$ & $n=543$ & $n=537$ & $\boldsymbol{P}$ \\
\hline Female gender & 58.4 & 56.9 & 56.1 & 52.1 & .20 & 45 & 58.5 & 62.7 & 59.3 & $<.0001$ \\
\hline Current smoker (yes) & 31.3 & 30.1 & 32.7 & 36.8 & .11 & 38.8 & 30.4 & 29.9 & 30.1 & .003 \\
\hline Alcohol drinking (yes) & 25.5 & 27.6 & 30.6 & 30.2 & .19 & 33.3 & 23.6 & 26.8 & 28.4 & .005 \\
\hline Married status & & & & & .52 & & & & & .038 \\
\hline Single & 3.3 & 3.2 & 1.4 & 2.7 & & 1.7 & 3 & 3.3 & 2.3 & \\
\hline Lived with spouse & 87.0 & 86.8 & 87.7 & 86.0 & & 90.1 & 87.9 & 85.8 & 84 & \\
\hline Divorced or separate & 9.8 & 10.0 & 10.8 & 11.3 & & 8.2 & 9.2 & 10.9 & 13.7 & \\
\hline Education level & & & & & .17 & & & & & .94 \\
\hline$<9 y$ & 93.7 & 94.7 & 91.4 & 93.6 & & 92.9 & 93.9 & 93.4 & 93.3 & \\
\hline$\geq 9 y$ & 6.3 & 5.3 & 8.6 & 6.4 & & 7.1 & 6.2 & 6.6 & 6.7 & \\
\hline Job status & & & & & .70 & & & & & $<.0001$ \\
\hline No job & 50.3 & 50.2 & 47.5 & 49.4 & & 35.5 & 47.8 & 54.8 & 58.7 & \\
\hline Blue collar & 32.5 & 33.3 & 32.2 & 33.9 & & 44.6 & 34.1 & 29.1 & 23.2 & \\
\hline White collar & 17.1 & 16.5 & 20.3 & 16.7 & & 19.9 & 18.1 & 16.1 & 18 & \\
\hline Regular exercise (yes) & 16.4 & 16.7 & 14.4 & 15.9 & .72 & 12.8 & 17.5 & 16.3 & 17.5 & .12 \\
\hline Family history of CHD & 9.6 & 9.8 & 11.2 & 9.5 & .78 & 10.4 & 10.3 & 9.5 & 10.2 & .96 \\
\hline Metabolic syndrome & 13.6 & 21.3 & 46.8 & 71.5 & $<.0001$ & 18.5 & 23.5 & 42.4 & 66.0 & $<.0001$ \\
\hline Diagnosed diabetes & 2.4 & 2.6 & 3.6 & 21.5 & $<.0001$ & 0.9 & 2.6 & 2.5 & 22.3 & $<.0001$ \\
\hline Age, mean, $y$ & 53.8 & 53.9 & 53.5 & 55.6 & .015 & 51.0 & 52.8 & 55.6 & 57.2 & $<.0001$ \\
\hline Body mass index, mean, $\mathrm{kg} / \mathrm{m}^{2}$ & 22.7 & 23.4 & 24.0 & 24.8 & $<.0001$ & 23.0 & 23.2 & 23.9 & 24.8 & $<.0001$ \\
\hline Systolic blood pressure, mean, $\mathrm{mm} \mathrm{Hg}$ & 122.3 & 123.9 & 127.2 & 130.8 & $<.0001$ & 121.9 & 122.1 & 126.7 & 133.1 & $<.0001$ \\
\hline $\mathrm{HDL}$ cholesterol, mean, mg/dL & 43.6 & 40.7 & 39.9 & 37.5 & $<.0001$ & 42.8 & 42.4 & 39.3 & 37.8 & $<.0001$ \\
\hline LDL cholesterol, mean, mg/dL & 124.2 & 128.9 & 128.6 & 138.3 & $<.0001$ & 120.1 & 127.2 & 132.3 & 140.8 & $<.0001$ \\
\hline
\end{tabular}

$H D L$, High density lipoprotein; LDL, low density lipoprotein. Values are expressed as percentage unless otherwise indicated.

from 1994-1995 to the end of 2005 (median 10.5, interquartile range 9.5-10.6 years), we included 2,165 individuals (953 men, 1,212 women) without CVD. Incident CVD included coronary heart disease and stroke cases. Incident coronary heart disease cases $(n=70)$ were defined as fatal coronary heart disease $(n=22)$ and hospitalization due to nonfatal myocardial infarction or percutaneous coronary intervention and coronary bypass surgery $(n=48)$. Nonfatal myocardial infarction and hospitalizations for percutaneous coronary intervention and coronary artery bypass graft were ascertained by the combined information from patient interviews and medical record review. Incident stroke cases $(n=100)$ were ascertained according to the following criteria: a sudden neurological deficit of vascular origin that lasted longer than 24 hours, with supporting evidence from the image study and medical records. The National Taiwan University Hospital Committee Review Board approved the study protocol.

\section{Measurement of glucose metabolism markers}

Analyses of glucose and insulin were prescribed previously. ${ }^{11}$ Briefly, after centrifugation by $1,500 \mathrm{~g}$ for 10 minutes, glucose levels were measured on supernatant by enzymatic assay (Merck 3389 commercial kit, Darmstadt, Germany) in a Eppendorf 5060 autoanalyzer (Eppendorf Corp., Hamburg, Germany). Plasma insulin level was determined using the ELISA method in which a reagent kit supplied by the Dako Co (Glostrup, Denmark) was used. We calculated the HOMA-insulin resistance (IR) ${ }^{5}$ and HOMA \%B. ${ }^{12}$ In addition, we estimated 2 markers for insulin sensitivity indices, QUICKI and ISI $_{0,120}$. QUICKI was calculated as inverse of sum logarithm fasting insulin and glucose. ${ }^{6}$ ISI $_{0,120}$ estimated the disposition of plasma glucose, given body weight and ambient insulin levels. ${ }^{7}$

We further grouped participants into 3 categories according to the American Diabetes Association (ADA) recommendation as follows: (1) postchallenge 2-hour glucose criteria-2-hour plasma glucose concentration of $200 \mathrm{mg} / \mathrm{dL}$ or more or on hypoglycemic medication as diabetes, $140-199 \mathrm{mg} / \mathrm{dL}$ for impaired glucose tolerance (IGT), $<140 \mathrm{mg} / \mathrm{dL}$ for normal glucose tolerance; (2) fasting glucose criteria alone-fasting plasma glucose $\geq 126 \mathrm{mg} / \mathrm{dL}$ or on hypoglycemic medication as diabetes, $110-125 \mathrm{mg} / \mathrm{dL}$ for impaired fasting glucose (IFG), and $<110 \mathrm{mg} / \mathrm{dL}$ for normal fasting glucose. 
Table II. Median levels, incidence rates, RRs (and 95\% Cls) of CVD events ( $n=166$ ) during 10 years of follow-up according to quartiles of baseline fasting glucose, postchallenge glucose and insulin resistance in 1994-1995 in the CCCC study

\begin{tabular}{|c|c|c|c|c|c|c|c|c|}
\hline Quartiles & 1 & 2 & $95 \% \mathrm{Cl}$ & 3 & $95 \% \mathrm{Cl}$ & 4 & $95 \% \mathrm{Cl}$ & $P$ trend \\
\hline Fasting glucose & 92 & 101 & & 111 & & 134 & & \\
\hline Rate (/1000 py) & 6.4 & 3.7 & & 9 & & 15.2 & & \\
\hline Model 1 & 1 & 0.55 & $0.31-0.98$ & 1.47 & $0.95-2.29$ & 2.21 & $1.46-3.33$ & $<.0001$ \\
\hline Model 2 & 1 & 0.55 & $0.31-0.97$ & 1.48 & $0.94-2.32$ & 2.09 & $1.37-3.19$ & $<.0001$ \\
\hline Model 3 & 1 & 0.49 & $0.26-0.90$ & 1.22 & $0.74-2.03$ & 1.74 & $1.06-2.86$ & .001 \\
\hline Postchallenge glucose & 87 & 113 & & 136 & & 196 & & \\
\hline Rate (/1000 py) & 4.7 & 5.7 & & 8.3 & & 15.3 & & \\
\hline Model 1 & 1 & 1.24 & $0.72-2.14$ & 1.50 & $0.90-2.50$ & 2.52 & $1.58-4.03$ & $<.0001$ \\
\hline Model 2 & 1 & 1.20 & $0.69-2.08$ & 1.45 & $0.87-2.42$ & 2.39 & $1.48-3.85$ & $<.0001$ \\
\hline Model 3 & 1 & 1.14 & $0.64-2.05$ & 1.24 & $0.72-2.14$ & 2.05 & $1.23-3.42$ & .001 \\
\hline Fasting insulin & 1.88 & 4.10 & & 6.60 & & 12.17 & & \\
\hline Rate (/1000 py) & 8.1 & 8.2 & & 7.6 & & 9.6 & & \\
\hline Model 1 & 1 & 1.23 & $0.79-1.91$ & 1.31 & $0.83-2.06$ & 1.62 & $1.05-2.50$ & .029 \\
\hline Model 2 & 1 & 1.21 & $0.77-1.90$ & 1.19 & $0.75-1.90$ & 1.43 & $0.90-2.27$ & .16 \\
\hline Model 3 & 1 & 1.03 & $0.64-1.65$ & 0.95 & $0.58-1.57$ & 0.97 & $0.58-1.60$ & .85 \\
\hline Postchallenge insulin & 6.045 & 17.1 & & 30.1 & & 60.49 & & \\
\hline Rate (/1000 py) & 7 & 8.1 & & 9.2 & & 9.1 & & \\
\hline Model 1 & 1 & 1.28 & $0.80-2.03$ & 1.32 & $0.84-2.09$ & 1.43 & $0.90-2.27$ & .66 \\
\hline Model 2 & 1 & 1.20 & $0.75-1.93$ & 1.29 & $0.81-2.04$ & 1.32 & $0.81-2.15$ & .87 \\
\hline Model 3 & 1 & 1.10 & $0.67-1.80$ & 1.13 & $0.69-1.84$ & 0.93 & $0.55-1.57$ & .57 \\
\hline HOMA-IR & 0.469 & 1.05 & & 1.79 & & 3.52 & & \\
\hline Rate (/1000 py) & 7.2 & 8.2 & & 7.2 & & 10.9 & & \\
\hline Model 1 & 1 & 1.37 & $0.87-2.15$ & 1.44 & $0.90-2.30$ & 1.99 & $1.29-3.07$ & .002 \\
\hline Model 2 & 1 & 1.36 & $0.86-2.15$ & 1.34 & $0.83-2.17$ & 1.82 & $1.14-2.91$ & .017 \\
\hline Model 3 & 1 & 1.31 & $0.80-2.15$ & 1.08 & $0.63-1.84$ & 1.36 & $0.80-2.30$ & .36 \\
\hline HOMA \%B & 14.74 & 33.4 & & 54.3 & & 95.00 & & \\
\hline Rate (/1000 py) & 10 & 9.6 & & 7.7 & & 6.3 & & \\
\hline Model 1 & 1 & 1.19 & $0.79-1.79$ & 1.07 & $0.70-1.64$ & 0.93 & $0.59-1.47$ & .31 \\
\hline Model 2 & 1 & 1.05 & $0.70-1.60$ & 0.94 & $0.60-1.45$ & 0.78 & $0.48-1.25$ & .11 \\
\hline Model 3 & 1 & 0.88 & $0.56-1.37$ & 0.81 & $0.51-1.28$ & 0.71 & $0.43-1.17$ & .11 \\
\hline QUICKI & 0.23 & 0.27 & & 0.32 & & 0.42 & & \\
\hline Rate (/1000 py) & 10.6 & 7.6 & & 8.2 & & 7.2 & & \\
\hline Model 1 & 1 & 0.78 & $0.51-1.19$ & 0.70 & $0.46-1.06$ & 0.52 & $0.34-0.80$ & .004 \\
\hline Model 2 & 1 & 0.81 & $0.52-1.24$ & 0.77 & $0.50-1.19$ & 0.57 & $0.36-0.91$ & .023 \\
\hline Model 3 & 1 & 0.88 & $0.56-1.38$ & 0.99 & $0.62-1.59$ & 0.77 & $0.45-1.30$ & .36 \\
\hline $\mid \mathrm{SI}_{0,120}$ & 0.1 & 0.16 & & 0.23 & & 0.39 & & \\
\hline Rate (/1000 py) & 13.5 & 8.5 & & 6.2 & & 5.9 & & \\
\hline Model 1 & 1 & 0.63 & $0.43-0.94$ & 0.53 & $0.34-0.82$ & 0.47 & $0.30-0.74$ & .37 \\
\hline Model 2 & 1 & 0.64 & $0.42-0.96$ & 0.56 & $0.35-0.87$ & 0.50 & $0.31-0.80$ & .36 \\
\hline Model 3 & 1 & 0.70 & $0.45-1.09$ & 0.68 & $0.41-1.12$ & 0.69 & $0.41-1.16$ & .36 \\
\hline
\end{tabular}

Incidence rates presented as per 1000 person years. Model 1 : adjusted for age groups (35-44, 45-54, 55-64, 65-74, $\geq 75$ years) and gender; model 2 : model 1 covariates plus body mass index $\left(<18,18-20.9,21-22.9,23-24.9\right.$, or $\left.\geq 25 \mathrm{~kg} / \mathrm{m}^{2}\right)$, smoking (yes/no or abstinence), current alcohol drinking (regular/no), marital status(single, married and living with spouse, or divorced and separate), education level (<9 years, at least 9 years), occupation (no work, labor, official or business), regular exercise habit (yes/no), and family history of coronary heart disease (yes/no); model 3: model 2 covariates plus the metabolic syndrome status (yes/no). py, Person years.

\section{Statistical analysis}

Participants were categorized on the basis of quartiles of postchallenge glucose levels. The CVD incidence rates were calculated as the number of cases divided by person-years of follow-up, stratified by various glucose and insulin resistance marker quartiles. We analyzed the associations between glucose and insulin resistance markers and risk of CVD by using Cox regression model, adjusted for potential confounding factors. Model 1 was adjusted for age groups (35-44, 45-54, 55-64, 65-74, $\geq 75$ years) and gender only. Model 2 included additional confounding factors including body mass index ( $<18,18-20.9,21-22.9,23-24.9$, or $\left.\geq 25 \mathrm{~kg} / \mathrm{m}^{2}\right)$, smoking (yes/no or abstinence), current alcohol drink- ing (regular/no), marital status (single, married and living with spouse, or divorced and separated), education level ( $<9$ years, at least 9 years), occupation (no work, labor, official, or business), regular exercise habit (yes/no), and family history of coronary heart disease (yes/no). In model 3, we additionally adjusted for the status of metabolic syndrome defined by the revised National Cholesterol Education Program (NCEP) criteria with Asian waist circumference criteria. ${ }^{13}$ To test for linear trend across categories, we used the median levels in quartiles as a continuous variable. In addition, we estimated the relative risks (RRs) associated with a change of one standard deviation in the glucose and insulin resistance variables. Furthermore, we used the 
Table III. Incidence rates (/ 1000 person-year) and RRs (and 95\% Cls) of CVD events during 10.5 years of follow-up according to ADA criteria of diabetes in 1994-1995 in the CCCC study

\begin{tabular}{|c|c|c|c|c|c|c|c|c|}
\hline Fasting glucose & Normal & IFG & $95 \% \mathrm{Cl}$ & Newly identified diabetes & $95 \% \mathrm{Cl}$ & Diagnosed diabetes & $95 \% \mathrm{Cl}$ & \\
\hline Number & 1276 & 466 & & 264 & & 158 & & \\
\hline Rate & 5.7 & 10.5 & & 11.2 & & 20.9 & & \\
\hline Model 1 & 1 & 1.86 & $1.27-2.71$ & 1.86 & $1.18-2.93$ & 3.13 & $2.00-4.90$ & $<.0001$ \\
\hline Model 2 & 1 & 1.87 & $1.28-2.75$ & 1.72 & $1.09-2.73$ & 3.01 & $1.90-4.75$ & $<.0001$ \\
\hline Model 3 & 1 & 1.58 & $1.02-2.46$ & 1.40 & $0.83-2.38$ & 2.78 & $1.67-4.64$ & .001 \\
\hline $\begin{array}{l}\text { Postchallenge } \\
\text { glucose }\end{array}$ & Normal & IGT & $95 \% \mathrm{Cl}$ & Newly identified diabetes & $95 \% \mathrm{Cl}$ & Diagnosed diabetes & $95 \% \mathrm{Cl}$ & $P$ trend \\
\hline Number & 1343 & 448 & & 149 & & 158 & & \\
\hline Rate & 5.8 & 9.3 & & 17.5 & & 20.9 & & \\
\hline Model 1 & 1 & 1.26 & $0.85-1.87$ & 2.37 & $1.47-3.83$ & 2.86 & $1.83-4.46$ & $<.0001$ \\
\hline Model 2 & 1 & 1.20 & $0.80-1.79$ & 2.32 & $1.42-3.78$ & 2.71 & $1.71-4.28$ & $<.0001$ \\
\hline Model 3 & 1 & 1.05 & $0.68-1.62$ & 1.88 & $1.11-3.18$ & 2.48 & $1.51-4.06$ & .0002 \\
\hline
\end{tabular}

Models as Table II.

area under the curve of receiver operative characteristics (ROC curve) to compare the discriminative ability of various glucose and insulin resistance markers. ${ }^{14}$ We examined the independent effects of fasting and postchallenge glucose levels on CVD outcomes by including 2 markers simultaneously in the model as continuous variables.

All statistical tests were 2-tailed and probability values $<.05$ were considered statistically significant. Analyses were performed with SAS version 9.1 (SAS Institute, Cary, NC) and Stata version 9.1 (Stata Corporation, College Station, TX).

\section{Results}

Participants with higher fasting glucose or postchallenge glucose levels were older, heavier, and had higher prevalence of metabolic syndrome and diagnosed diabetes (Table I). Participants with higher postchallenge glucose had similar characteristics in terms of obesity, lifestyle factors, and socioeconomic status as those with higher fasting glucose.

Table II showed the RRs of CVD events during 10.5 years of follow-up across quartiles of markers baseline. After adjustment for cardiovascular risk factors and the metabolic syndrome, both fasting and postchallenge glucose were significantly associated with CVD (RR in the highest quartile vs the lowest quartile $1.74,95 \%$ confidence interval $[\mathrm{CI}] \mathbf{1 . 0 6 - 2 . 8 6}$ for fasting glucose; RR in highest quartile 2.05, 95\% CI 1.23-3.42 for postchallenge glucose). Other markers, such as HOMA-IR and QUICKI also showed significant associations with CVD in multivariate model (RR 1.82, 95\% CI 1.14-2.91 for HOMA-IR; RR 0.57 , 95\% CI 0.36-0.91 for QUICKI), but these associations were attenuated and became nonsignificant after additional adjustment for the presence of metabolic syndrome. The RRs of various glucose and insulin measurements did not change appreciably even after controlling for systolic blood pressure, high-density lipoprotein (HDL) cholesterol and low-density lipoprotein (LDL) cholesterol in the models. With regard to one standard deviation change, fasting and postchallenge glucose levels were only significant predictors for CVD events in the multivariate adjusted model (RR 1.25, 95\% CI 1.11-1.40 for fasting glucose; RR 1.29, 95\% CI 1.16-1.43 for postchallenge glucose).

The patterns of the above associations were similar when incident coronary heart disease or stroke cases were analyzed separately (data not shown). In addition, exclusion of subjects with diagnosed diabetes at baseline did not substantially alter the findings. Table III showed the RRs of CVD events according to the ADA definitions of glucose status. After adjustment for cardiovascular risk factors and the metabolic syndrome, the newly identified diabetes defined by postchallenge glucose had a stronger association with CVD than that defined by fasting glucose. However, IFG was significantly associated with CVD (RR 1.58, 95\% CI 1.02-2.46), but IGT was not (RR $1.05,95 \%$ CI 0.68-1.62).

To compare the predictive values of various glucose and insulin resistance markers, we estimated the ROC curve for each marker separately. The results showed that postchallenge and fasting glucose had similar areas of ROC curve $(0.648,95 \%$ CI $0.581-0.715$ for postchallenge glucose; 0.645 , 95\% CI 0.575-0.715 for fasting glucose). We conducted the likelihood ratio test and found postchallenge glucose had the highest $\chi^{2}$ value (71.5) than fasting glucose (21.2).

In the final model, both fasting and postchallenge glucose were significantly associated with CHD risk after mutual adjustment: the RRs comparing extreme quartiles 
Table IV. Relative risks and $95 \% \mathrm{Cls}$ of the highest quartiles from multivariate models with combination of fasting glucose, postchallenge glucose and another insulin resistance marker, after adjustment for confounding factors

\begin{tabular}{|c|c|c|c|c|c|c|c|}
\hline Marker & $\mathbf{R R}$ & $95 \% \mathrm{Cl}$ & $P$ trend & Marker & $\mathbf{R R}$ & $95 \% \mathrm{Cl}$ & $P$ trend \\
\hline Fasting glucose & 1.61 & $1.01-2.58$ & .003 & Postchallenge glucose & 1.72 & $1.02-2.89$ & .015 \\
\hline Fasting glucose & 2.09 & $1.36-3.24$ & $<.0001$ & Fasting insulin & 1.10 & $0.68-1.77$ & .79 \\
\hline Fasting glucose & 2.01 & $1.31-3.09$ & $<.0001$ & Postchallenge insulin & 1.14 & $0.70-1.86$ & .65 \\
\hline Fasting glucose & 2.01 & $1.26-3.19$ & $<.0001$ & HOMA-IR & 1.19 & $0.71-2.00$ & .64 \\
\hline Fasting glucose & 2.02 & $1.31-3.10$ & $<.0001$ & HOMA \%B & 0.94 & $0.57-1.52$ & .77 \\
\hline Fasting glucose & 2.01 & $1.27-3.19$ & $<.0001$ & QUICKI & 0.87 & $0.52-1.45$ & .57 \\
\hline Fasting glucose & 1.83 & $1.14-2.94$ & .001 & $I S I_{0,120}$ & 0.72 & $0.43-1.21$ & .28 \\
\hline Postchallenge glucose & 2.34 & $1.44-3.80$ & $<.0001$ & Fasting insulin & 1.18 & $0.73-1.92$ & .62 \\
\hline Postchallenge glucose & 2.71 & $1.54-4.76$ & $<.0001$ & Postchallenge insulin & 0.75 & $0.42-1.34$ & .29 \\
\hline Postchallenge glucose & 2.20 & $1.34-3.59$ & .000 & HOMA-IR & 1.43 & $0.87-2.36$ & .22 \\
\hline Postchallenge glucose & 2.42 & $1.50-3.90$ & $<.0001$ & HOMA \%B & 0.83 & $0.51-1.36$ & .34 \\
\hline Postchallenge glucose & 2.19 & $1.34-3.59$ & .001 & QUICKI & 0.73 & $0.44-1.20$ & .21 \\
\hline Postchallenge glucose & 2.73 & $1.19-6.27$ & .003 & $\mid \mathrm{SI}_{0,120}$ & 1.18 & $0.51-2.72$ & .60 \\
\hline
\end{tabular}

of fasting and postchallenge glucose were 1.61 (95\% CI $1.01-2.58, P$ trend $=.003)$ and $1.72(95 \%$ CI $1.02-2.89$, $P$ trend $=.015)$, respectively (Table IV). Further adjustment for metabolic syndrome only slightly attenuated these associations.

\section{Discussion}

In this prospective cohort of middle-aged to older ethnic Chinese, higher levels of fasting and postchallenge glucose were significantly associated with increased risk of CVD. Insulin resistance indices (HOMA and QUICKI) were also associated with CVD risk, but these associations became nonsignificant after further adjustment for the metabolic syndrome.

Several previous studies showed postchallenge glucose was more strongly associated with CVD than fasting glucose. ${ }^{15-19}$ The prospective Diabetes Epidemiology: Collaborative analysis Of Diagnostic Criteria in Europe (DECODE) study demonstrated a linear relationship between postchallenge hyperglycemia and coronary heart disease risk, but an independent relationship with fasting hyperglycemia was only found in high glucose level. ${ }^{15}$ Subsequent analyses of the DECODE study found stronger association with cardiovascular and all-cause mortality for postchallenge glucose than for fasting glucose during approximately 9 years of follow-up. ${ }^{16}$ In a small Italian study, postprandial, but not fasting blood glucose significantly predicted cardiovascular events in diabetic patients during 5 years of follow-up. ${ }^{17}$

Other studies, however, suggested that fasting glucose was as predictive of morbidity and mortality as postchallenge glucose. In the Baltimore Longitudinal Study of Aging, ${ }^{20}$ both fasting plasma glucose and IGT were significantly associated increased risk of mortality during 13.4 years of follow-up. A meta-analysis of 38 reports demonstrated a dose-response positive relationship between fasting and postchallenge glucose and
CVD incident and mortality, with the RR slightly higher for the postchallenge glucose. The pooled RRs comparing the highest with the lowest categories were 1.58 (95\% CI 1.19-2.10) for postchallenge and 1.33 (95\% CI 1.06-1.67) for fasting glucose. ${ }^{21}$ Our data suggested that very low fasting glucose levels might increase risk of CVD, consistent with a U-shape relationship association between fasting glucose and the risk of CVD. ${ }^{22}$ This relationship may be explained by elevated catecholamine and decreased potassium levels in hypoglycemia that were associated with cardiac ischemia and poor prognosis. ${ }^{23}$ Moreover, our findings showed that the risk associated with higher fasting glucose decreased appreciably after adjusting for the metabolic syndrome. This may result from overcontrol because an elevated fasting glucose is a component of metabolic syndrome.

Previous study suggested that fasting hyperglycemia was associated with $\beta$ cell dysfunction, whereas postchallenge hyperglycemia tended to be more strongly related to insulin resistance, higher blood pressure, obesity, and dyslipidemia rates. ${ }^{3}$ Previous studies on the associations between IFG and IGT and CVD risk have been inconclusive. ${ }^{24,25}$ In our study, according to the ADA criteria, newly identified diabetes by postchallenge glucose had higher RR than that defined by fasting glucose criteria. On the other hand, IFG was more strongly associated with CVD risk than IGT. These discrepant results may result from relatively small number of cases in those categories.

Our results support the hypothesis that postchallenge glucose levels provide additional information beyond measuring fasting glucose, possibly reflecting increased peripheral tissue insulin resistance. Although fasting glucose is now used as a standard diagnostic criterion for diabetes, the oral glucose tolerance test still provides important information for predicting CVD risk. ${ }^{26}$ In clinical practice and in lifestyle and pharmacological 
interventions, the postchallenge glucose should still be emphasized as a target to reduce the diabetes incidence and CVD risk. ${ }^{8}$ Furthermore, the C-statistics for both fasting and postchallenge glucose were similar (0.645 and 0.648 , respectively), suggesting that either one has independent discriminatory power in predicting future cardiovascular events. Therefore, it seems that either fasting glucose or postchallenge glucose but not both would be sufficient in risk-stratification of Chinese adults.

To our knowledge, this is the first extensive investigation on various glucose and insulin resistance markers and risk of CVD in Chinese. Studies from other Asian populations have demonstrated that postchallenge glucose may be related to CVD events, ${ }^{27,28}$ but the head-tohead comparison of postchallenge and fasting glucose is not available. Because of the prospective cohort design, the baseline measurements of all cohort members were unlikely to be affected by storage and laboratory issues that might be raised in some nested case-control studies. In addition, the use of a community-based population could reduce the possibility of selection bias. We also included important socioeconomic status and lifestyle factors in the models to control for potential confounding factors.

Our study had several potential limitations. First, the number of incident cases of CVD events was relatively small, even with 10.5 years follow-up. This would reduce the power to detect the subtle differences between various glucose and insulin markers and make some of the RR estimates unstable. Second, because glucose and insulin levels were measured only once, our results might be prone to intraindividual variations, which might have attenuated our results. Finally, the study results may not be generalizable to other ethnic groups.

In conclusion, our data suggest that fasting and postchallenge glucose concentrations are statistically significant independent predictors of CVD risk in a Chinese population. Our data indicate that postchallenge glucose, a measurement of peripheral tissue insulin resistance status, should be taken into consideration in assessing CVD risk in Asian populations. Because of only moderate correlation coefficients between postchallenge glucose and other glucose and insulin resistance markers, postchallenge glucose can provide useful information for comprehensive evaluation of CVD risk in Asian populations.

We thank the participants in the Chin-Shan community for their participation.

\section{References}

1. DeFronzo RA, Ferrannini E, Simonson DC. Fasting hyperglycemia in non-insulin-dependent diabetes mellitus: contributions of excessive hepatic glucose production and impaired tissue glucose uptake. Metabolism 1989;38:387-95
2. Unwin N, Shaw J, Zimmet P, et al. Impaired glucose tolerance and impaired fasting glycaemia: the current status on definition and intervention. Diabet Med 2002;19:708-23.

3. Davies M, Raymond NT, Day JL, et al. Impaired glucose tolerance and fasting hyperglycaemia have different characteristics. Diabet Med 2000;17:433-40.

4. Meyer C, Pimenta W, Woerle HJ, et al. Different mechanisms for impaired fasting glucose and impaired postprandial glucose tolerance in humans. Diabetes Care 2006;29:1909-14.

5. Matthws DR, Hosker JP, Rudenski AS, et al. Homeostasis model assessment: insulin resistance and ?cell function from fasting plasma glucose and insulin concentrations in man. Diabetologia 1985;28: $412-9$.

6. Katz A, Nambi SS, Mather K, et al. Quantitative insulin sensitivity check index: a simple, accurate method for assessing insulin sensitivity in humans. J Clin Endocrinol Metab 2000;85: 2402-10.

7. Gutt M, Davis $C L$, Spitzer $S B$, et al. Validation of the insulin sensitivity index (ISI $(0,120))$ : comparison with other measures. Diabetes Res Clin Pract 2000;47:177-84.

8. Yamagishi S, Nakamura K, Takeuchi M. Inhibition of postprandial hyperglycemia by acarbose is a promising therapeutic strategy for the treatment of patients with the metabolic syndrome. Med Hypotheses 2005;65:152-4.

9. Chien KL, Hsu HC, Sung FC, et al. Hyperuricemia as a risk factor on cardiovascular events in Taiwan: The Chin-Shan Community Cardiovascular Cohort study. Atherosclerosis 2005; 183:147-55.

10. Lee YT, Lin RS, Sung FC, et al. Chin-Shan Community Cardiovascular Cohort in Taiwan: baseline data and five-year follow-up morbidity and mortality. Journal of Clinical Epidemiology 2000;53: 836-46.

11. Chien KL, Lee YT, Sung FC, et al. Hyperinsulinemia and related atherosclerotic risk factors in the population at cardiovascular risk: a community-based study. Clinical Chemistry 1999;45:838-46.

12. Wallace TM, Levy JC, Matthews DR. Use and abuse of HOMA modeling. Diabetes Care 2004;27:1487-95.

13. Tan CE, Ma S, Wai $D$, et al. Can we apply the National Cholesterol Education Program Adult Treatment Panel definition of the metabolic syndrome to Asians? Diabetes Care 2004;27: 1182-1186.

14. Greiner M, Pfeiffer D, Smith RD. Principles and practical application of the receiver-operating characteristic analysis for diagnostic tests. Prev Vet Med 2000;45:23-41.

15. The DECODE study group, European Diabetes Epidemiology Group. Diabetes Epidemiology: Collaborative analysis Of Diagnostic criteria in Europe. Glucose tolerance and mortality: comparison of WHO and American Diabetes Association diagnostic criteria. Lancet 1999;354: 617-21.

16. Decode Study Group. Glucose tolerance and cardiovascular mortality: comparison of fasting and 2-hour diagnostic criteria. Arch Intern Med 2001;161:397-405.

17. Cavalot F, Petrelli A, Traversa M, et al. Postprandial blood glucose is a stronger predictor of cardiovascular events than fasting blood glucose in type 2 diabetes mellitus, particularly in women: lessons from the San Luigi Gonzaga Diabetes Study. J Clin Endocrinol Metab 2006;91:813-9.

18. Qiao $Q$, Pyorala K, Pyorala $M$, et al. Two-hour glucose is a better risk predictor for incident coronary heart disease and cardiovascular mortality than fasting glucose. Eur Heart J 2002;23: 1267-75.

19. Tominaga $M$, Eguchi $H$, Manaka $H$, et al. Impaired glucose tolerance is a risk factor for cardiovascular disease, but not impaired fasting 
glucose. The Funagata Diabetes Study. Diabetes Care 1999;22: 920-4.

20. Sorkin JD, Muller DC, Fleg JL, et al. The relation of fasting and 2-h postchallenge plasma glucose concentrations to mortality: data from the Baltimore Longitudinal Study of Aging with a critical review of the literature. Diabetes Care 2005;28:2626-32.

21. Coutinho M, Gerstein HC, Wang Y, et al. The relationship between glucose and incident cardiovascular events. A metaregression analysis of published data from 20 studies of 95,783 individuals followed for 12.4 years. Diabetes Care 1999;22:233-40.

22. Wei M, Gibbons LW, Mitchell TL, et al. Low fasting plasma glucose level as a predictor of cardiovascular disease and all-cause mortality. Circulation 2000;101:2047-52.

23. Svensson AM, McGuire DK, Abrahamsson P, et al. Association between hyper- and hypoglycaemia and 2 year all-cause mortality risk in diabetic patients with acute coronary events. Eur Heart J 2005; 26:1255-61.
24. Blake DR, Meigs JB, Muller DC, et al. Impaired glucose tolerance, but not impaired fasting glucose, is associated with increased levels of coronary heart disease risk factors: results from the Baltimore Longitudinal Study on Aging. Diabetes 2004;53:2095-100.

25. Hanefeld M, Temelkova-Kurktschiev T, Schaper F, et al. Impaired fasting glucose is not a risk factor for atherosclerosis. Diabet Med 1999; 16:212-8.

26. Li CL, Tsai ST, Chou P. Comparison of metabolic risk profiles between subjects with fasting and 2-hour plasma glucose impairment: The Kinmen Study. J Clin Epidemiol 2002;55:19-24.

27. Wang JJ, Yuan SY, Zhu LX, et al. Effects of impaired fasting glucose and impaired glucose tolerance on predicting incident type 2 diabetes in a Chinese population with high post-prandial glucose. Diabetes Res Clin Pract 2004;66:183-91.

28. Lawes CM, Parag V, Bennett DA, et al. Blood glucose and risk of cardiovascular disease in the Asia Pacific region. Diabetes Care 2004;27:2836-42. 